\title{
Dynamic Analysis of a Timoshenko Beam Subjected to an Accelerating Mass Using Spectral Element Method
}

\author{
Guangsong Chen, Linfang Qian, and Qiang Yin \\ College of Mechanical Engineering, Nanjing University of Science and Technology, Nanjing, Jiangsu 210094, China \\ Correspondence should be addressed to Linfang Qian; Lfqian@vip.163.com
}

Received 27 October 2012; Accepted 28 April 2013; Published 27 February 2014

Academic Editor: Reza Jazar

Copyright ( 2014 Guangsong Chen et al. This is an open access article distributed under the Creative Commons Attribution License, which permits unrestricted use, distribution, and reproduction in any medium, provided the original work is properly cited.

\begin{abstract}
This paper presents formulations for a Timoshenko beam subjected to an accelerating mass using spectral element method in time domain (TSEM). Vertical displacement and bending rotation of the beam were interpolated by Lagrange polynomials supported on the Gauss-Lobatto-Legendre (GLL) points. By using GLL integration rule, the mass matrix was diagonal and the dynamic responses can be obtained efficiently and accurately. The results were compared with those obtained in the literature to verify the correctness. The variation of the vibration frequencies of the Timoshenko and moving mass system was researched. The effects of inertial force, centrifugal force, Coriolis force, and tangential force on a Timoshenko beam subjected to an accelerating mass were investigated.
\end{abstract}

\section{Introduction}

Dynamic response of structures subjected to a moving force or moving mass is an important issue in engineering problems. For example, the trains have experienced great advances characterized by increasingly higher speeds and weights of vehicles. As a result, the dynamic response, as well as stresses, can be significantly higher than that before or static loads. The problem arose from the observations is a structure subjected to moving masses. Many researchers studied these problems and many studies are presented in the literatures. For examples, references [1-3] assuming the moving load to be a moving force have given some analytical solutions. Abu-Hilal [4] studied the dynamic response of a double Euler-Bernoulli beam due to a moving constant load. Fryba [5] extensively analyzed the solution of moving loads on structures. Rao [6] gave a detailed analysis of the vibration of a beam excited by a moving oscillator using a perturbation method. Zibdeh and Juma [7] presented the dynamic response of a rotating beam subjected to a random moving load using analytical and numerical methods. Akin and Mofid [8] investigated the dynamic behavior of Bernoulli-Euler beams carrying a moving mass with different boundary conditions using analytical-numerical method, and achievements by other researchers are presented in literatures [9-14].
The studies mentioned are based on Bernoulli-Euler beam, while the moment of inertia and shear deformation should be taken into account when ratio of the height to span is large. References [15-20] studied the dynamic response of Timoshenko beams subject to moving force. Ross [21] studied the problem of a viscoelastic Timoshenko beam subjected to a step-loading using the Laplace transform method. Katz et al. [22] solved the dynamic response of a rotating shaft subjected to a moving load with constant velocity using the modal analysis method and an integral transformation method. Yavari et al. [23] determined the dynamic behavior of Timoshenko beams due to a moving mass using the discrete element technique (DET). Lee [24] obtained the dynamic responses of a Timoshenko beam subjected to a moving mass using the AMM.

Two different kinds of spectral element method (SEM) have been developed to analyse various problems of engineering, namely, spectral element method in frequency domain (FSEM) and TSEM. The FSEM proposed by Doyle [25] is accurate and suited for simple $1 \mathrm{D}$ or $2 \mathrm{D}$ problems [2628 ], while 3D problems or complex geometry are analyzed difficultly by this method and it does not work for nonlinear problems. TSEM is first used by Patera [29] to fluid dynamics; it has characteristics of spectral analysis and finite element method, thus, it is highly accurate and suited for complex 
geometry. This method has been successfully applied to many problems, that is, wave, fluid, seismology and acoustics [3035].

In this paper, a Timoshenko beam spectral element has been developed on the basis of Legendre polynomials-based spectral finite element. The beam is discretized into a very small number of elements with ten degrees of freedom each. The equations of motion in matrix form for a Timoshenko beam due to a moving mass are derived by using the Hamilton principle. The shape functions of the vertical displacement and the bending rotation for a Timoshenko beam element are formulated by employing Lagrange interpolation supported on the GLL points. The element mass matrix, stiffness matrix, and damping matrix are obtained by GLL integration rule. The effects of inertial forces are considered by the added mass, stiffness, and damping matrix. By assembling element matrices and element nodal vectors, respectively, the global equations of motion for a Timoshenko beam subjected to a moving mass are obtained. The vibration frequencies of the Timoshenko and a moving mass system were analyzed; in addition, the effects of the various forces considering the inertia of the mass will be investigated in detail. The presented formulations can be applied to solve the eigenvalue problem and dynamic responses for a Timoshenko beam with various boundary conditions by direct integration using generalized$\omega_{1}$ method.

\section{Theory and Formulation}

A uniform Timoshenko beam acted on by a moving mass $m$ with a variable speed $v(t)$ and acceleration $a(t)$ along the beam is shown in Figure 1. A coordinate system is assumed to be fixed in the inertial frame with the $x$-axis parallel to the undeformed longitudinal axis of the beam and the $y$ axis pointing vertically downward in reverse direction as the gravitational acceleration $g$.

According to the Timoshenko's beam theory, the deformed beam can be described by the rotation of the across-area and the shear deformation [36]. The relation of the all rotation, section rotation, and shear strain is as follows:

$$
\frac{\partial y}{\partial x}=\varphi+\gamma
$$

where $\varphi$ and $\gamma$ are, respectively, the bending rotation and shear strain.

As the shear strain $\gamma \neq 0$, the curvature of the Timoshenko beam is different from that of the Euler-Bernoulli beam and it can be expressed as follows:

$$
R=-\frac{\mathrm{d} \varphi}{\mathrm{d} x}
$$

where $R$ is the curvature of the Timoshenko beam.

The Timoshenko beam is discretized into a very small number of spectral elements with equal length, $l$ each. Every beam element consists of five nodes; each node has two degrees of freedom, that is, vertical displacement $y$ and section rotation $\varphi$. The nodes are not uniformly distributed on the element. These nodes, denoted by $\xi_{j} \in[-1,1], j=$ $1, \ldots, k$, are the GLL points which are the $(k)$ roots of

$$
\left(1-\xi^{2}\right) L_{k-1}^{\prime}(\xi)=0
$$

where $L_{k-1}^{\prime}(\xi)$ is the derivative of the Legendre polynomial of degree $(k-1)$.

Figure 2 shows the $n$-th beam element on which the moving mass applies, at time $t$. The position $x \in\left[\begin{array}{ll}0 & l\end{array}\right]$ of the arbitrary point in the element can be transfered to the local coordinate along the axis of the beam element $\xi \in[-1,1]$ by

$$
\xi=\frac{2}{l} x-1
$$

where $l$ is the length of the element. The vertical displacement $y$ and section rotation $\varphi$ of an arbitrary point on the beam element can be expressed as follows:

$$
\begin{aligned}
& y=\left[\begin{array}{lllll}
N_{1} & N_{2} & N_{3} & N_{4} & N_{5}
\end{array}\right]\left\{\begin{array}{l}
y_{1}^{n} \\
y_{2}^{n} \\
y_{3}^{n} \\
y_{4}^{n} \\
y_{5}^{n}
\end{array}\right\}=[\mathbf{N}]\left\{\mathbf{y}^{n}\right\}, \\
& \varphi=\left[\begin{array}{lllll}
N_{1} & N_{2} & N_{3} & N_{4} & N_{5}
\end{array}\right]\left\{\begin{array}{c}
\varphi_{1}^{n} \\
\varphi_{2}^{n} \\
\varphi_{3}^{n} \\
\varphi_{4}^{n} \\
\varphi_{5}^{n}
\end{array}\right\}=[\mathbf{N}]\left\{\boldsymbol{\varphi}^{n}\right\},
\end{aligned}
$$

where $\left\{\mathbf{y}^{n}\right\}$ and $\left\{\boldsymbol{\varphi}^{n}\right\}$ are, respectively, the element nodal displacement and section rotation vector. The shape functions $N_{i}$ which are the orthogonal shape functions that denote Lagrange interpolation supported on the $k$ GLL points can be expressed as follows:

$$
N_{i}(\xi)=\prod_{j=1, j \neq i}^{k} \frac{\xi-\xi_{j}}{\xi_{i}-\xi_{j}}
$$

where $k$ is the number of nodes. The equation of motion for the Timoshenko beam and moving mass system can be obtained by using the Hamilton principle, expressed as follows:

$$
\delta \int_{t_{1}}^{t_{2}} L \mathrm{~d} t=0,
$$

where $L$ is Lagrange function; it can be represented by

$$
L=T+U+W
$$

where $T, U$, and $W$ are, respectively, the kinetic energy, potential energy, and work done by external force. $\delta$ is the 


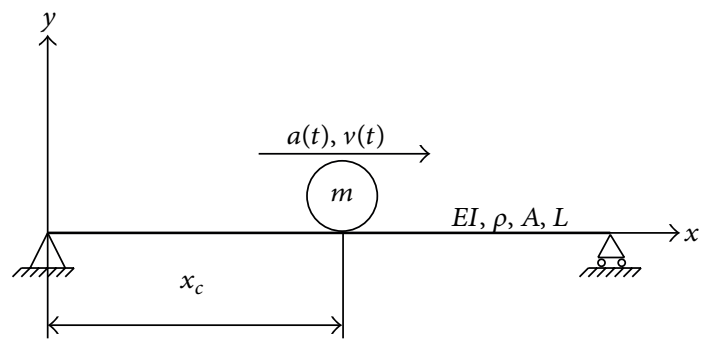

FIGURE 1: Schematic figure of a Timoshenko beam and a moving mass system.

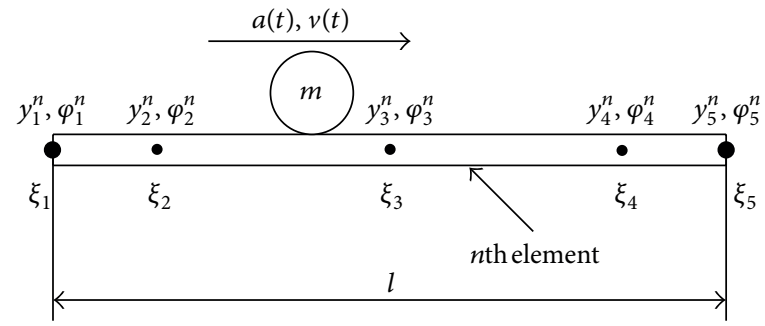

FIgUre 2: The $n$th Timoshenko beam spectral element with an accelerating mass.

variation operator; $t_{1}$ and $t_{2}$ are any instance. The kinetic energy of the Timoshenko beam element is given by

$$
\begin{aligned}
T & =\frac{1}{2} \rho A \int_{0}^{l}\left(\frac{\partial y(x, t)}{\partial t}\right)^{2} \mathrm{~d} \xi+\frac{1}{2} \rho I \int_{0}^{l}\left(\frac{\partial \varphi(x, t)}{\partial t}\right)^{2} \mathrm{~d} \xi \\
& =\frac{1}{2} \rho A \int_{-1}^{1} \dot{y}(\xi, t)^{2} \frac{l}{2} \mathrm{~d} \xi+\frac{1}{2} \rho I \int_{-1}^{1} \dot{\varphi}(\xi, t)^{2} \frac{l}{2} \mathrm{~d} \xi
\end{aligned}
$$

where $\rho$ is the density of the beam, $A$ is the cross-sectional area, $I$ is the second order moment of area, and the superposed dot stands for differentiation with respect to time. The potential energy of the Timoshenko beam element is expressed as follows:

$$
\begin{aligned}
U= & \frac{1}{2} \int_{0}^{l} E I R^{2} \mathrm{~d} x+\frac{1}{2} \int_{0}^{l} \frac{G A}{\kappa}\left(\frac{\partial y}{\partial x}-\varphi\right)^{2} \mathrm{~d} x \\
= & \frac{1}{2} \int_{-1}^{1} \frac{l}{2} E I\left(\frac{2}{l}\right)^{2} \varphi^{\prime}(\xi)^{2} \mathrm{~d} \xi \\
& +\frac{1}{2} \int_{-1}^{1} \frac{l}{2} \frac{G A}{\kappa} \frac{2}{l}\left(y^{\prime}(\xi)-\varphi\right)^{2} \mathrm{~d} \xi,
\end{aligned}
$$

where $E$ is the Young's modulus, $G$ is the shear modulus, and $\kappa$ is the shear coefficient depending on the shape of the crosssection; the prime symbol $\left(^{\prime}\right)$ denotes differentiation with respect to $\xi$.
When the moving mass is located at $x=x_{c}$, the virtual work done by the moving force is represented by

$$
\begin{aligned}
\delta W= & \int_{0}^{l}\left(g-\frac{\partial^{2} y}{\partial t^{2}}+2 v \frac{\partial^{2} y}{\partial x \partial t}+v^{2} \frac{\partial^{2} y}{\partial x^{2}}+a \frac{\partial y}{\partial x}\right) \\
& \times \delta y(\xi) \delta\left(x-x_{c}\right) \mathrm{d} x \\
= & m \int_{-1}^{1} \frac{l}{2}\left(g-\ddot{y}(\xi)+2 v \frac{2}{l} \dot{y}^{\prime}(\xi)\right. \\
& \left.+v^{2}\left(\frac{2}{l}\right)^{2} y^{\prime \prime}(\xi)+a \frac{2}{l} y^{\prime}(\xi)\right) \\
& \times \delta y(\xi) \frac{\delta\left(\xi+1-(2 / l) x_{c}\right)}{l / 2} \mathrm{~d} \xi,
\end{aligned}
$$

where $g$ is the gravitational acceleration. $\delta\left(\xi+1-(2 / l) x_{c}\right)$ represents the Dirac delta function at $\xi=(2 / l) x_{c}-1$. By introducing (5), the expressions of the differentiations with respect to local coordinate $\xi$ and time $t$ for $y$ and $\varphi$ in (9)-(11) can be written as

$$
\begin{aligned}
\ddot{\mathbf{y}} & =[\mathbf{N}]\left\{\ddot{\mathbf{y}}^{n}\right\}, \\
\ddot{\boldsymbol{\varphi}} & =[\mathbf{N}]\left\{\ddot{\boldsymbol{\varphi}}^{n}\right\}, \\
\mathbf{y}^{\prime} & =\left[\mathbf{N}^{\prime}\right]\left\{\mathbf{y}^{n}\right\}, \\
\boldsymbol{\varphi}^{\prime} & =\left[\mathbf{N}^{\prime}\right]\left\{\boldsymbol{\varphi}^{n}\right\}, \\
\mathbf{y}^{\prime \prime} & =\left[\mathbf{N}^{\prime \prime}\right]\left\{\mathbf{y}^{n}\right\}, \\
\dot{\mathbf{y}}^{\prime} & =\left[\mathbf{N}^{\prime}\right]\left\{\dot{\mathbf{y}}^{n}\right\} .
\end{aligned}
$$

By substituting (12a)-(12f) into (8) and (8) into (7), respectively, and using the Hamilton principle, the equation 
of motion for a Timoshenko beam element that acted on by a moving mass in matrix form can be expressed as

$$
\mathbf{M}^{e} \ddot{\mathbf{q}}^{e}+\mathbf{C}^{e} \dot{\mathbf{q}}^{e}+\mathbf{K}^{e} \mathbf{q}^{e}=\mathbf{F}^{e}
$$

where $\mathbf{M}^{e}, \mathbf{C}^{e}$, and $\mathbf{K}^{e}$ are the mass, damping, and stiffness matrices of the Timoshenko beam element, respectively, $\mathbf{F}^{e}$ is the equivalent nodal force vector of the element, and $\ddot{\mathbf{q}}^{e} \dot{\mathbf{q}}^{e}$, and $\mathbf{q}$ denote the nodal acceleration, velocity, and displacement vector of the element, respectively.

Where

$$
\mathbf{q}=\left\{\begin{array}{c}
\mathbf{y}^{e} \\
\boldsymbol{\varphi}^{e}
\end{array}\right\}
$$

$\mathbf{M}^{e}, \mathbf{C}^{e}$, and $\mathbf{K}^{e}$ can be expressed as

$$
\begin{gathered}
\mathbf{M}^{e}=\mathbf{M}_{T}^{e}+\mathbf{M}_{f}^{e}, \\
\mathbf{C}^{e}=\mathbf{C}_{f}^{e}, \\
\mathbf{K}^{e}=\mathbf{K}_{b}^{e}+\mathbf{K}_{s}^{e}+\mathbf{K}_{f 1}^{e}+\mathbf{K}_{f 2}^{e},
\end{gathered}
$$

where $\mathbf{M}_{f}^{e}, \mathbf{C}_{f}, \mathbf{K}_{f 1}^{e}$, and $\mathbf{K}_{f 2}^{e}$ are, respectively, added mass, damping, and stiffness matrix coming from the moving load; the matrices $\mathbf{M}_{T}^{e}, \mathbf{M}_{f}^{e}, \mathbf{C}_{f}^{e}, \mathbf{K}_{b}^{e}, \mathbf{K}_{s}^{e}, \mathbf{K}_{f 1}^{e}, \mathbf{K}_{f 2}^{e}$, and $\mathbf{F}^{e}$ are matrices defined as

$$
\begin{gathered}
\mathbf{M}_{T}^{e}=\int_{-1}^{1} \mathbf{N}^{T}\left[\begin{array}{cc}
\rho A & 0 \\
0 & \rho I
\end{array}\right] \mathbf{N} \mathrm{d} \xi \\
\mathbf{M}_{f}^{e}=m \int_{-1}^{1} \mathbf{N}^{T} \mathbf{N} \delta\left(\xi+1-\frac{2}{l} x_{c}\right) \mathrm{d} \xi \\
\mathbf{C}_{f}^{e}=2 m v \int_{-1}^{1} \mathbf{N}^{T} \mathbf{N}^{\prime} \delta\left(\xi+1-\frac{2}{l} x_{c}\right) \mathrm{d} \xi \\
\mathbf{K}_{b}^{e}=E I \int_{-1}^{1} \mathbf{B}_{b}^{T} \mathbf{B}_{b} \mathrm{~d} \xi \\
\mathbf{K}_{f 1}^{e}=m v^{2} \int_{-1}^{1} \mathbf{N}^{T} \mathbf{N}^{\prime \prime} \delta\left(\xi+1-\frac{2}{l} x_{c}\right) \mathrm{d} \xi \\
\mathbf{K}_{f 2}^{e}=m a \int_{0}^{1} \mathbf{N}^{T} \mathbf{N}_{s} \mathrm{~d} \xi \\
\mathbf{F}^{e}=\int_{-1}^{1} m g \mathbf{N}^{T} \delta\left(\xi+1-\frac{2}{l} x_{c}\right) \mathrm{d} \xi
\end{gathered}
$$

in which

$$
\begin{aligned}
& \mathbf{N}=\left[\begin{array}{cccccccccc}
N_{1} & 0 & N_{2} & 0 & N_{3} & 0 & N_{4} & 0 & N_{5} & 0 \\
0 & N_{1} & 0 & N_{2} & 0 & N_{3} & 0 & N_{4} & 0 & N_{5}
\end{array}\right], \\
& \mathbf{N}^{\prime}=\frac{2}{l}\left[\begin{array}{cccccccccc}
N_{1}^{\prime} & 0 & N_{2}^{\prime} & 0 & N_{3}^{\prime} & 0 & N_{4}^{\prime} & 0 & N_{5}^{\prime} & 0 \\
0 & N_{1}^{\prime} & 0 & N_{2}^{\prime} & 0 & N_{3}^{\prime} & 0 & N_{4}^{\prime} & 0 & N_{5}^{\prime}
\end{array}\right],
\end{aligned}
$$

$$
\begin{aligned}
\mathbf{N}^{\prime \prime}= & \left(\frac{2}{l}\right)^{2} \\
& \times\left[\begin{array}{cccccccccc}
N_{1}^{\prime \prime} & 0 & N_{2}^{\prime \prime} & 0 & N_{3}^{\prime \prime} & 0 & N_{4}^{\prime \prime} & 0 & N_{5}^{\prime \prime} & 0 \\
0 & N_{1}^{\prime \prime} & 0 & N_{2}^{\prime \prime} & 0 & N_{3}^{\prime \prime} & 0 & N_{4}^{\prime \prime} & 0 & N_{5}^{\prime \prime}
\end{array}\right],
\end{aligned}
$$

$\mathbf{B}_{b}$

$$
=\left[\begin{array}{llllllllll}
0 & -\frac{2}{l} N_{1}^{\prime} & 0 & -\frac{2}{l} N_{2}^{\prime} & 0 & -\frac{2}{l} N_{3}^{\prime} & 0 & -\frac{2}{l} N_{4}^{\prime} & 0 & -\frac{2}{l} N_{5}^{\prime}
\end{array}\right]
$$

$$
\begin{aligned}
\mathbf{B}_{s}=\left[-\frac{2}{l} N_{1}^{\prime}-N_{1}^{\prime}-\frac{2}{l} N_{2}^{\prime}-N_{2}^{\prime}-\frac{2}{l} N_{3}^{\prime}-N_{3}^{\prime}\right. \\
\left.-\frac{2}{l} N_{4}^{\prime}-N_{4}^{\prime}-\frac{2}{l} N_{5}^{\prime}-N_{5}^{\prime}\right],
\end{aligned}
$$

where the superscript $T$ denotes the transpose. The matrices $\mathbf{M}_{T}^{e}, \mathbf{K}_{b}^{e}$, and $\mathbf{K}_{s}^{e}$ should be calculated numerically by GLL integral which is different from Gauss integral; the integral nodes are the GLL nodes which contain the boundary nodes. It can be written as follows:

$$
\begin{gathered}
\mathbf{M}_{T}^{e}=\int_{-1}^{1} \mathbf{N}^{T}\left[\begin{array}{cc}
\rho A & 0 \\
0 & \rho I
\end{array}\right] \mathbf{N d} \xi=\sum_{i=1}^{n} \omega_{i} \mathbf{N}^{T}\left(\xi_{i}\right)\left[\begin{array}{cc}
\rho A & 0 \\
0 & \rho I
\end{array}\right] \mathbf{N}\left(\xi_{i}\right) \\
\mathbf{K}_{b}^{e}=E I \int_{-1}^{1} \mathbf{B}_{b}^{T} \mathbf{B}_{b} \mathrm{~d} \xi=E I \sum_{i=1}^{n} \omega_{i} \mathbf{B}_{b}^{T}\left(\xi_{i}\right) \mathbf{B}_{b}\left(\xi_{i}\right), \\
\mathbf{K}_{s}^{e}=\frac{G A}{k} \int_{-1}^{1} \mathbf{B}_{s}^{T} \mathbf{B}_{s} \mathrm{~d} \xi=\frac{G A}{k} \sum_{i=1}^{n} \omega_{i} \mathbf{B}_{s}^{T}\left(\xi_{i}\right) \mathbf{B}_{s}\left(\xi_{i}\right)
\end{gathered}
$$

where $E I$ and $G A$ are the bending stiffness and shear stiffness, respectively; $\rho A$ and $\rho I$ are the inertia properties coefficient; weights $\omega_{i}$ are determined numerically by

$$
\omega_{i}=\frac{2}{k(k+1)} \cdot \frac{1}{\left[L_{k}\left(x_{i}\right)\right]^{2}}, \quad i=0,1, \ldots, k .
$$

By assembling element matrices and element nodal vectors, respectively, the global equations of motion for a Timoshenko beam subjected to a moving mass can be obtained as follows:

$$
M \ddot{q}+C \dot{q}+K q=F,
$$

where the matrices $\mathbf{M}, \mathbf{C}$, and $\mathbf{K}$ are the global mass, damping, and stiffness matrices, respectively, of the Timoshenko beam, the vectors $\ddot{\mathbf{q}}, \dot{\mathbf{q}}$, and $\mathbf{q}$ are the global nodal acceleration, 
velocity, and displacement vectors, respectively, and the vector $\mathbf{F}$ is the equivalent nodal force. If the added matrices of the mass and the equivalent force vector are zero, undamped natural frequencies and vibration node-shape of the beam are obtained from homogenous solution of (20). For this case, (20) reduces to

$$
\mathbf{M q}+\mathbf{K q}=\mathbf{0} .
$$

The solution of (21) can be expressed as

$$
\begin{gathered}
\mathbf{q}=\mathbf{A} e^{\mathrm{j} \omega t}, \\
\ddot{\mathbf{q}}=-\omega^{2} \mathbf{A} e^{\mathrm{j} \omega t} .
\end{gathered}
$$

Introducing (22a) and (22b) into (21) gives

$$
\left(\mathbf{K}-\omega^{2} \mathbf{M}\right) \boldsymbol{\Phi} \mathrm{e}^{\mathrm{j} \omega t}=\mathbf{0} .
$$

As $\Phi \mathrm{e}^{\mathrm{j} \omega t} \neq 0$, the solution of (23) exits only if the determinant of $\left(\mathbf{K}-\omega^{2} \mathbf{M}\right)$ is zero.

Consider the following:

$$
\operatorname{det}\left(\mathbf{K}-\omega^{2} \mathbf{M}\right)=0
$$

By solving (24) and (23), the frequencies and the corresponding vectors of the dynamic system can be obtained.

In order to obtain the dynamic response of the Timoshenko beam and the moving mass system, the generalized$\alpha$ time integration method is used. It has more stability and lower numerical dissipation and numerical dispersion. In the generalized- $\alpha$ method, (20) is transformed to

$$
\begin{gathered}
\mathbf{K}^{*}=a_{0} \mathbf{M}\left(1-\alpha_{m}\right)+a_{1} \mathbf{C}\left(1-\alpha_{f}\right)+\mathbf{K}\left(1-\alpha_{f}\right), \\
\mathbf{F}^{*}=\mathbf{F}\left[\left(1-\alpha_{f}\right) t_{i+1}+\alpha_{f} t_{i}\right]-\alpha_{f} \mathbf{K q}_{i} \\
+\mathbf{C}\left[a_{1}\left(1-\alpha_{f}\right) \mathbf{u}_{i}+\alpha_{4}\left(1-\alpha_{f}\right) \dot{\mathbf{u}}_{i}\right. \\
\left.+a_{5}\left(1-\alpha_{f}\right) \ddot{\mathbf{u}}_{i}-\alpha_{f} \dot{\mathbf{u}}_{i}\right] \\
+\mathbf{M}\left[a_{0}\left(1-\alpha_{m}\right) \mathbf{q}_{i}+a_{2}\left(1-\alpha_{m}\right) \dot{\mathbf{q}}_{i}\right. \\
\left.+a_{3}\left(1-\alpha_{m}\right) \ddot{\mathbf{q}}_{i}-\alpha_{m} \ddot{\mathbf{q}}_{i}\right]
\end{gathered}
$$

where $a_{0}, a_{1}, a_{2}, a_{3}, a_{4}, a_{5}, a_{6}$, and $a_{7}$ are, respectively, the same as that in Newmark- $\beta$ method; that is,

$$
\begin{array}{ll}
a_{0}=\frac{1}{\beta \Delta t^{2}}, & a_{1}=\frac{\gamma}{\beta \Delta t}, \\
a_{2}=\frac{1}{\beta \Delta t}, & a_{3}=\frac{1}{2 \beta}-1 \\
a_{4}=\frac{\gamma}{\beta}-1, & a_{5}=\frac{\Delta t}{2}\left(\frac{\gamma}{\beta}-2\right), \\
a_{6}=\Delta t(1-\gamma), & a_{7}=\gamma \Delta t,
\end{array}
$$

where $\alpha_{m}, \alpha_{f}, \beta$, and $\gamma$ are the parameters which can control the precision and stability of the algorithm. In this study, the following values are used: $\alpha_{f}=\alpha_{m}=1 / 2, \beta=1 / 4$, and $\gamma=$ $1 / 2$; the details of the generalized- $\alpha$ method can be referred to in [37].

The updated equations for the displacement velocity and acceleration vectors are expressed as

$$
\begin{gathered}
\mathbf{K}^{*} \mathbf{q}_{i+1}=\mathbf{F}^{*}, \\
\ddot{\mathbf{q}}_{i+1}=a_{0}\left(\mathbf{q}_{i+1}-\mathbf{q}_{i}\right)-a_{2} \dot{\mathbf{q}}_{i}-a_{3} \ddot{\mathbf{q}}_{i}, \\
\dot{\mathbf{q}}_{i+1}=\dot{\mathbf{q}}_{i}+a_{6} \ddot{\mathbf{q}}_{i}+a_{7} \ddot{\mathbf{q}}_{i+1} .
\end{gathered}
$$

\section{Numerical Calculations and Analysis}

To illustrate this method and verify its effectiveness and correctness, examples 1 and 2 are solved; the solutions obtained by the proposed method are compared with those obtained by other methods (including exact analytical solutions). Example 3 is solved to present the variation of the vibration frequencies of the system during the mass moves on the beam. Example 4 is solved to present the effects of inertial force, centrifugal force, Coriolis force, and tangential force on a Timoshenko beam subjected to an accelerating mass.

3.1. Example 1. Three types of prismatic Timoshenko beams considering the natural vibration problems are solved by the present method. The parameters of the beams are the same with different boundaries, simple-simple, clamped-free, and clamped-simple. The properties of the beam are modulus of elasticity $E=2.1 \times 10^{11} \mathrm{~N} / \mathrm{m}^{2}$, modulus of rigidity $G=3 E / 8$, density $\rho=7850 \mathrm{~kg} / \mathrm{m}^{3}$, beam length $L=0.4 \mathrm{~m}$, the cross section in the form of a rectangle with width $b=0.02 \mathrm{~m}$ and height $h=0.08 \mathrm{~m}$, and cross-sectional shape factor $\kappa=2 / 3$.

To test the convergence and validity of the method, the first nine-order natural frequencies obtained by presented method are compared with the analytical solution presented by Huang [38]. Fifth order of the spectral element is deployed to solve the problem, and the numbers of the beam spectral elements are from 1 to 16 . The natural frequencies determined by the proposed method and the exact value calculated on the basis of [38] and Ruta [39] are shown in Table 1 to Table 3.

Tables 1, 2, and 3 show that the first nine frequencies of the Timoshenko beam are convergent by using 16 fiveorder Timoshenko beam spectral elements. As the excellent characteristic of the SEM that the mass matrix is diagonal because of the choice of Lagrange interpolation function supported on the GLL points in conjunction with the GLL integration rule, the values of the natural frequencies are equal to those of the exact.

As the proposed method has the advantage of the finite elements and the spectral method, it can analyse free vibration problem with various boundary conditions and good accuracy.

3.2. Example 2. For the purpose of verification, a Timoshenko beam and a moving mass system neglecting the damping effect with boundary condition simple-simple were considered. The beam is discretized to 16 elements by fiveorder Timoshenko beam spectral element. The dynamic 
TABLE 1: Free vibration frequencies for simple-simple Timoshenko beam.

\begin{tabular}{|c|c|c|c|c|c|c|c|c|c|}
\hline & $\omega_{1}$ & $\omega_{2}$ & $\omega_{3}$ & $\omega_{4}$ & $\omega_{5}$ & $\omega_{6}$ & $\omega_{7}$ & $\omega_{8}$ & $\omega_{9}$ \\
\hline$n=4$ & 6838.8338 & 23190.888 & 43444.615 & 64925.614 & 86738.326 & 108481.00 & 111981.29 & 120647.23 & 129920.78 \\
\hline$n=8$ & 6838.8336 & 23190.827 & 43443.498 & 64939.222 & 86711.057 & 108431.78 & 111981.29 & 120647.23 & 130004.36 \\
\hline$n=12$ & 6838.8336 & 23190.827 & 43443.493 & 64939.186 & 86710.905 & 108431.36 & 111981.29 & 120647.23 & 130003.65 \\
\hline$n=16$ & 6838.8336 & 23190.827 & 43443.493 & 64939.185 & 86710.899 & 108431.34 & 111981.29 & 120647.23 & 130003.61 \\
\hline Exact [38] & 6838.8336 & 23190.827 & 43443.493 & 64939.185 & 86710.889 & 108431.34 & 111981.29 & 120647.23 & 130003.61 \\
\hline
\end{tabular}

TABlE 2: Free vibration frequencies for clamped-free Timoshenko beam.

\begin{tabular}{|c|c|c|c|c|c|c|c|c|c|}
\hline & $\omega_{1}$ & $\omega_{2}$ & $\omega_{3}$ & $\omega_{4}$ & $\omega_{5}$ & $\omega_{6}$ & $\omega_{7}$ & $\omega_{8}$ & $\omega_{9}$ \\
\hline$n=4$ & 2529.4927 & 13279.912 & 31045.083 & 50829.910 & 71550.753 & 91995.694 & 110969.19 & 119289.42 & 131460.96 \\
\hline$n=8$ & 2529.4927 & 13279.905 & 31044.792 & 50825.847 & 71565.121 & 91995.100 & 110976.56 & 119244.54 & 131607.53 \\
\hline$n=12$ & 2529.4927 & 13279.905 & 31044.791 & 50825.835 & 71565.050 & 91994.834 & 110976.00 & 119244.58 & 131606.55 \\
\hline$n=16$ & 2529.4927 & 13279.905 & 31044.791 & 50825.843 & 71565.047 & 91994.824 & 110975.98 & 119244.57 & 131606.52 \\
\hline Exact [38] & 2529.4927 & 13279.905 & 31044.791 & 50825.843 & 71565.047 & 91994.824 & 110975.98 & 119244.57 & 131606.52 \\
\hline
\end{tabular}

TABLE 3: Free vibration frequencies for clamped-simple Timoshenko beam.

\begin{tabular}{lccccccccc}
\hline & $\omega_{1}$ & $\omega_{2}$ & $\omega_{3}$ & $\omega_{4}$ & $\omega_{5}$ & $\omega_{6}$ & $\omega_{7}$ & $\omega_{8}$ & $\omega_{9}$ \\
\hline$n=4$ & 9741.9478 & 26150.351 & 45547.079 & 66197.391 & 87399.691 & 108645.85 & 114294.62 & 128761.33 & 131483.10 \\
$n=8$ & 9741.9469 & 26150.251 & 45545.516 & 66212.036 & 87376.814 & 108601.58 & 114295.46 & 128739.40 & 131611.56 \\
$n=12$ & 9741.9469 & 26150.251 & 45545.510 & 66211.995 & 87376.651 & 108601.16 & 114295.44 & 128739.41 & 131610.66 \\
$n=16$ & 9741.9469 & 26150.251 & 45545.510 & 66211.993 & 87376.644 & 108601.14 & 114295.44 & 128739.40 & 131610.63 \\
Reference [39] & 9741.9469 & 26150.251 & 45545.510 & 66211.993 & 87376.644 & 108601.14 & 114295.44 & 128739.40 & 131610.63 \\
\hline
\end{tabular}

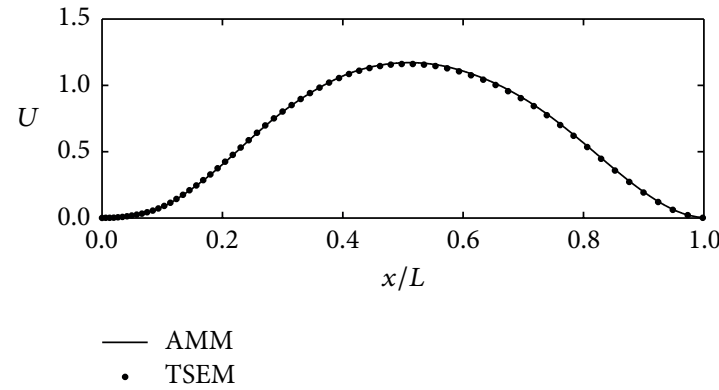

(a)

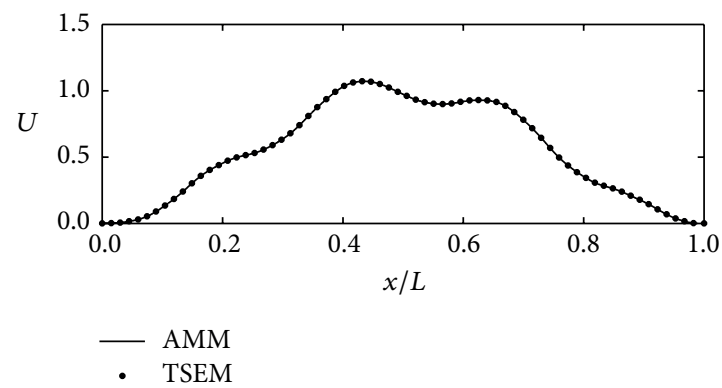

(b)

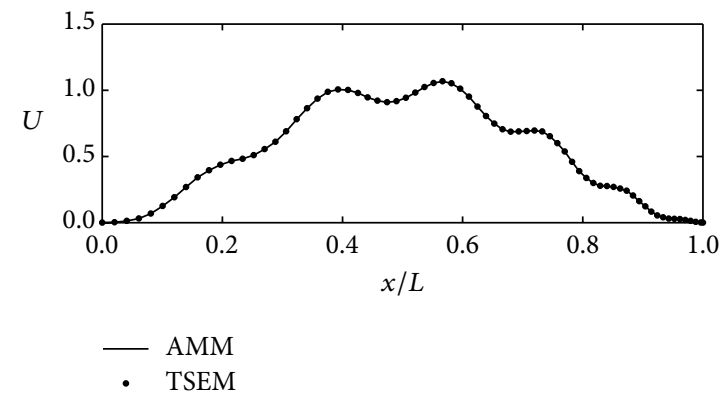

(c)

FIgURE 3: Normalized displacement under the equivalent moving loads with the initial speed given by $\tau=0.11 . \beta=0.03$. (a) $\lambda=1$, (b) $\lambda=0$, and (c) $\lambda=-0.05$. 


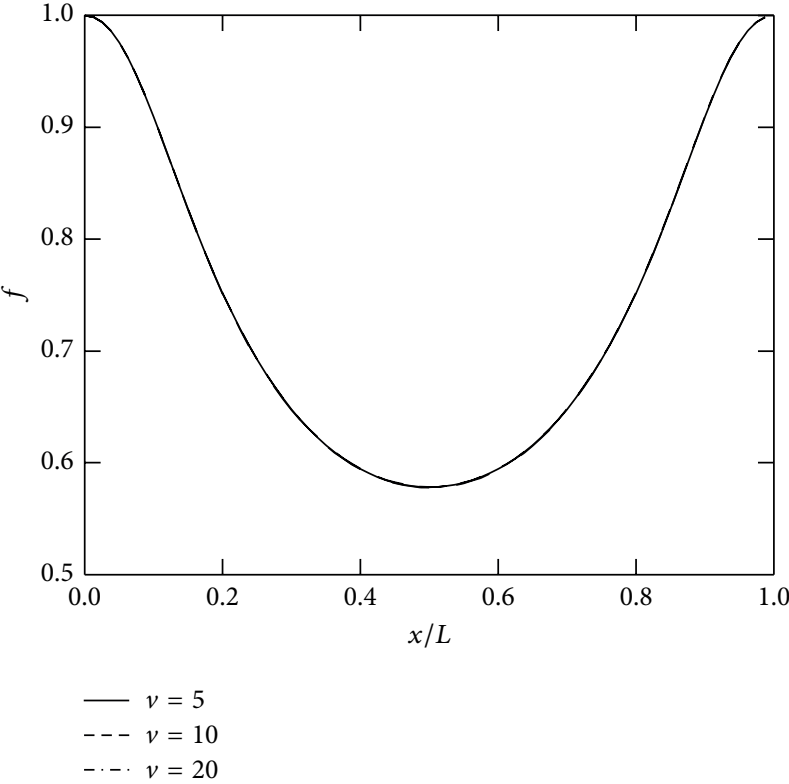

(a)

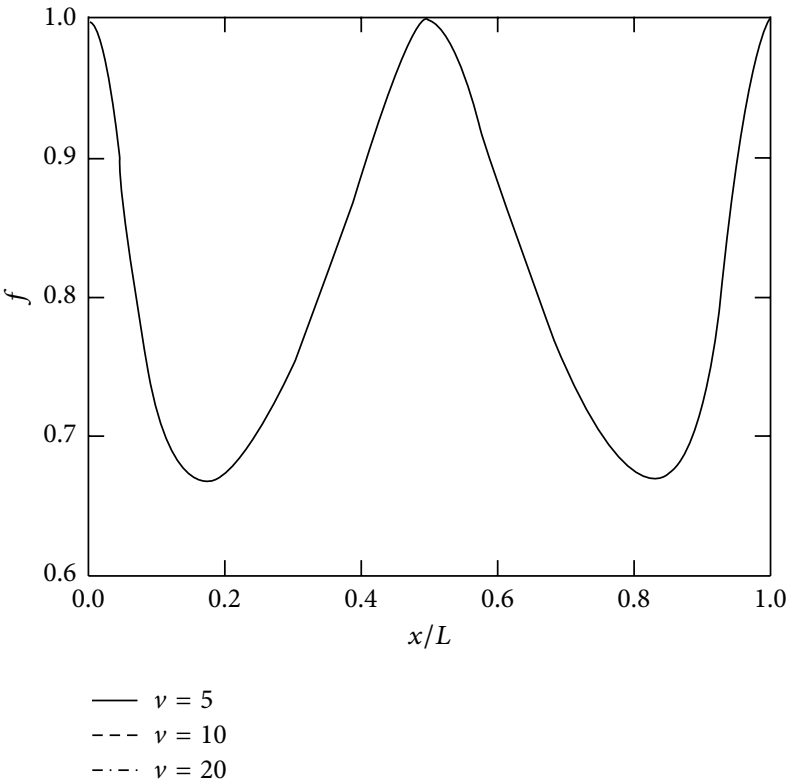

(b)

FIGURE 4: The first two normalized natural frequencies of simple-simple Timoshenko beam subjected to a moving mass with different velocities.

response of the system is obtained by using generalized- $\alpha$ method with $\alpha_{f}=\alpha_{m}=1 / 2, \beta=1 / 4$, and $\gamma=1 / 2$. The same parameters and material properties of the Timoshenko beam as defined in Lee [24] are used in the numerical simulation, that is, $L=1 \mathrm{~m}, E=2.07 \times 10^{11} \mathrm{~N} / \mathrm{m}^{2}, G=7.76 \times 10^{10} \mathrm{~N} / \mathrm{m}^{2}$, $\kappa=0.9$, and $\rho=7700 \mathrm{~kg} / \mathrm{m}^{3}$. The cross-sectional area $A$ of the beam is computed from the radius of gyration $r_{0}$ defined by a nondimensional parameter (Rayleigh's coefficient) $\beta=$ $r_{0} \pi / L$. The prescribed travelling speed of the moving mass, $v$, is similarly defined by a nondimensional parameter given by $\tau=v / v_{\mathrm{cr}}$, where $v_{\mathrm{cr}}=(\pi / L) \sqrt{E I / \rho A}$. The acceleration of the moving mass $a$ is similarly defined by a nondimensional parameter $\lambda=a m L / E I$.

The vertical displacement of the beam is normalized by $y_{\mathrm{st}}$, defining normalized displacement $U=y / y_{\mathrm{st}}$, where $y_{\text {st }}=M g L^{3} / 48 E I$ is the beam static displacement at midpoint when a load $M g$ is applied at the same point for an Euler-Bernoulli beam.

Figure 3 shows the normalized displacements under the moving mass by $\lambda=1, \lambda=0$, and $\lambda=-0.05$, respectively. The numerical results for the displacements agreed very much with the reported results by AMM using ten-term assumed functions [24].

3.3. Example 3. With only the equivalent force vector that is zero, the natural frequencies of the beam and moving masses system are obtained. For this case, we rewrite (20) as

$$
\mathbf{A} \dot{\mathbf{p}}+\mathbf{B} \mathbf{p}=\mathbf{0}
$$

where

$$
\begin{gathered}
\mathbf{A}=\left[\begin{array}{cc}
\mathbf{M} & \mathbf{0} \\
\mathbf{0} & -\mathbf{K}
\end{array}\right], \quad \mathbf{B}=\left[\begin{array}{cc}
\mathbf{0} & \mathbf{M} \\
\mathbf{M} & \mathbf{C}
\end{array}\right], \\
\mathbf{p}=\left\{\begin{array}{l}
\dot{\mathbf{q}} \\
\mathbf{q}
\end{array}\right\}
\end{gathered}
$$

And the eigenvalue problem relatives to (25) can be expressed as

$$
\begin{gathered}
\mathbf{A} \mathbf{u}=\lambda \mathbf{B} \mathbf{u}, \\
\mathbf{v}^{T} \mathbf{A}=\lambda \mathbf{v}^{T} \mathbf{B},
\end{gathered}
$$

where $\lambda$ is the vibration frequency and $\mathbf{u}$ and $\mathbf{v}$ are the right and left vector of the system, respectively.

The properties and parameters of the beam are the same as Example 1. Figures 4, 5, 6, 7, 8, and 9 show the first two normalized frequencies of the same Timoshenko beam subjected to a moving mass with different boundary conditions. It has a constant mass with $m=10 \mathrm{~kg}$ and different initial speed of the mass with $v=5,10,20 \mathrm{~m} / \mathrm{s}$ in Figure 4 to Figure 6, while in Figure 7 to Figure 9 the initial speed is the same with $v=10 \mathrm{~m} / \mathrm{s}$ and the masses are $m=2,5$ and $10 \mathrm{~kg}$, respectively. the acceleration is $a=0 \mathrm{~m} / \mathrm{s}^{2}$. The vibration frequencies $\omega_{s}$ normalized by the natural frequencies $\omega$ of the beam with same boundary are denoted by $f$ defined by $f=\omega_{s} / \omega$.

Figure 4 to Figure 9 indicate the natural frequencies of the beam and a moving mass system that are modified periodicity during the mass moving on the beam, and the number of the periods is equal to the order of the frequencies; it also shows that frequencies are not more than those of the Timoshenko 

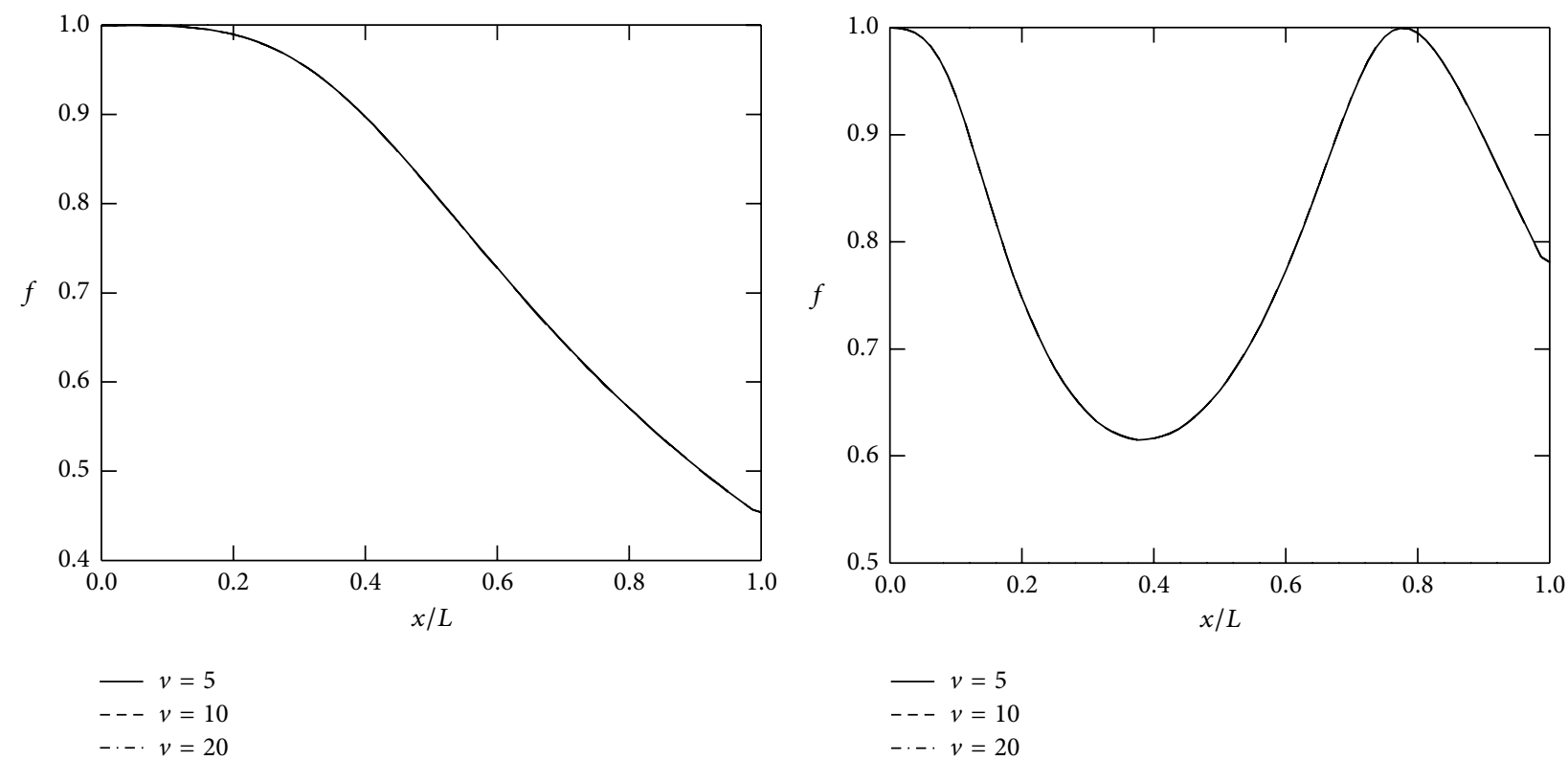

(a)

(b)

Figure 5: The first two normalized natural frequencies of clamped-free Timoshenko beam subjected to a moving mass with different velocities.
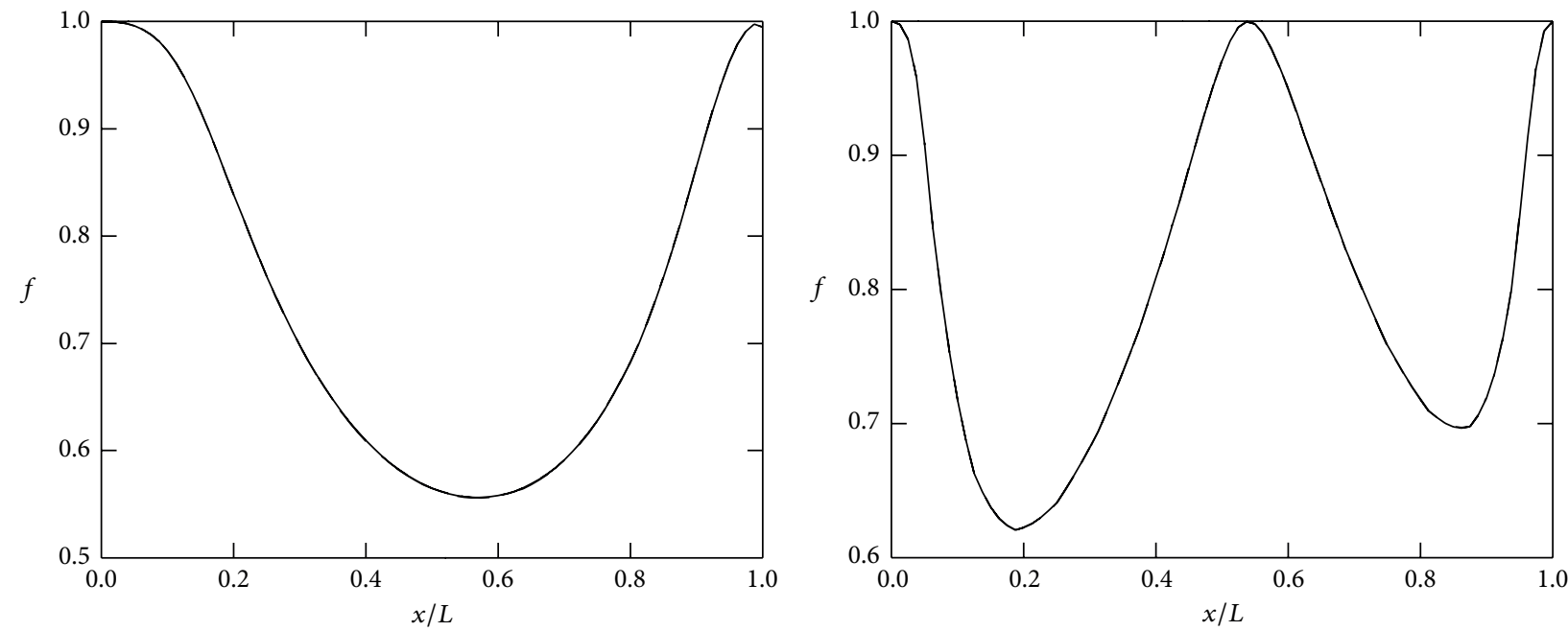

$$
\begin{aligned}
-v & =5 \\
---v & =10 \\
--v & =20
\end{aligned}
$$$$
-v=5
$$$$
--v=10
$$$$
\text { -. . } v=20
$$

(a)

(b)

FIGURE 6: The first two normalized natural frequencies of clamped-simple Timoshenko beam subjected to a moving mass with different velocities.

beam. It can also be seen in Figure 3 to Figure 9 that the frequencies are not sensitive to the velocity of the mass, while they can be sensitive to the mass, as the velocity affects the added damping and stiffness matrices, and the added stiffness matrix is smaller than that of the beam with bending stiffness $E I$ and shear stiffness $G A$, while the added mass affected with the inertia of the mass is corresponding to the inertia property coefficient $\rho A$ of the mass matrix in the motion equations.
3.4. Example 4. To analyse the effect of the different forces considering the inertia of the moving mass, the Timoshenko beam and a moving mass system are introduced with the same parameters as Example 2. The inertial force, Coriolis force, centrifugal force, and tangential force at the moving mass point are, respectively, shown in Figure 10.

Motion equations show that all the forces are the important members of the loads considering the effect of the 


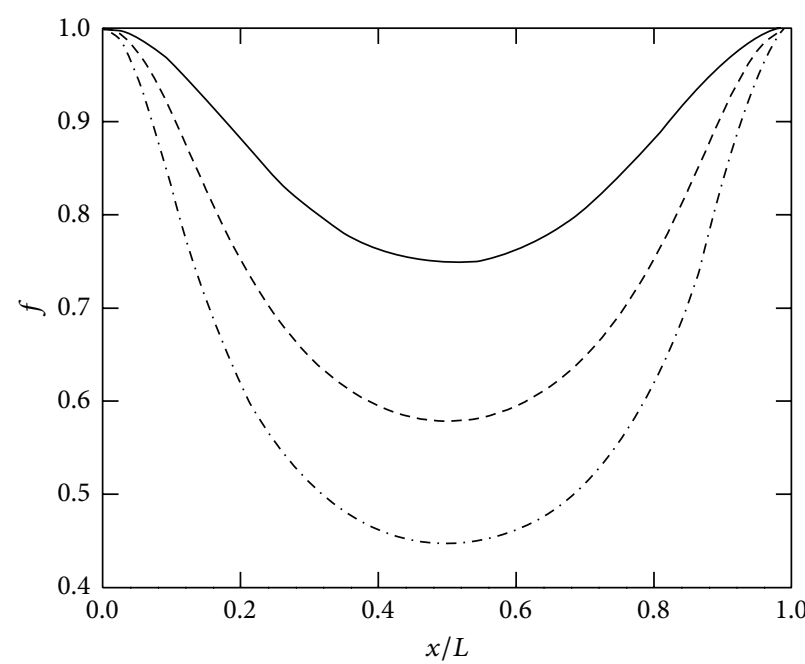

$\begin{aligned} m & =2 \\ --m & =5 \\ -\cdots & m=10\end{aligned}$

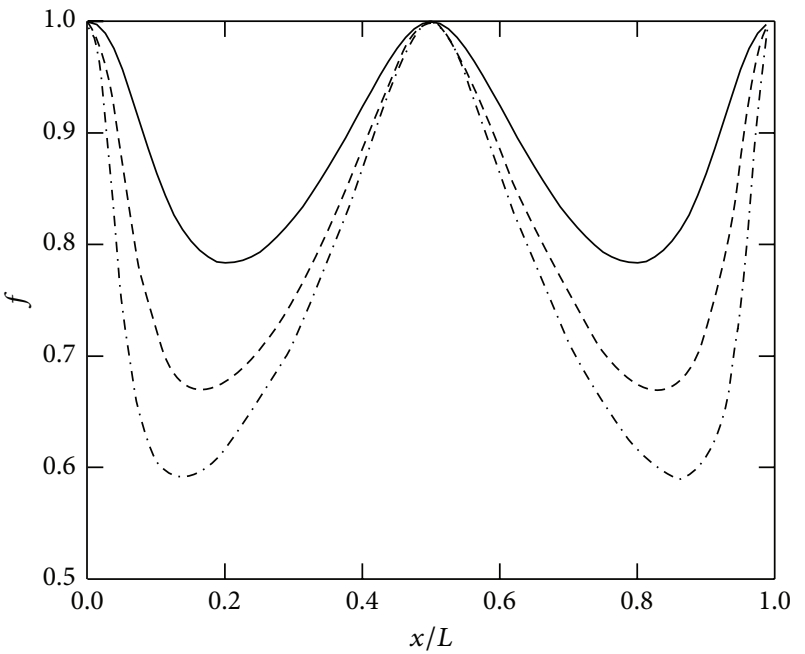

$-m=2$

$--m=5$

-... $m=10$

(a)

(b)

FIGURE 7: The first two normalized natural frequencies of simple-simple Timoshenko beam subjected to a moving mass with different masses.

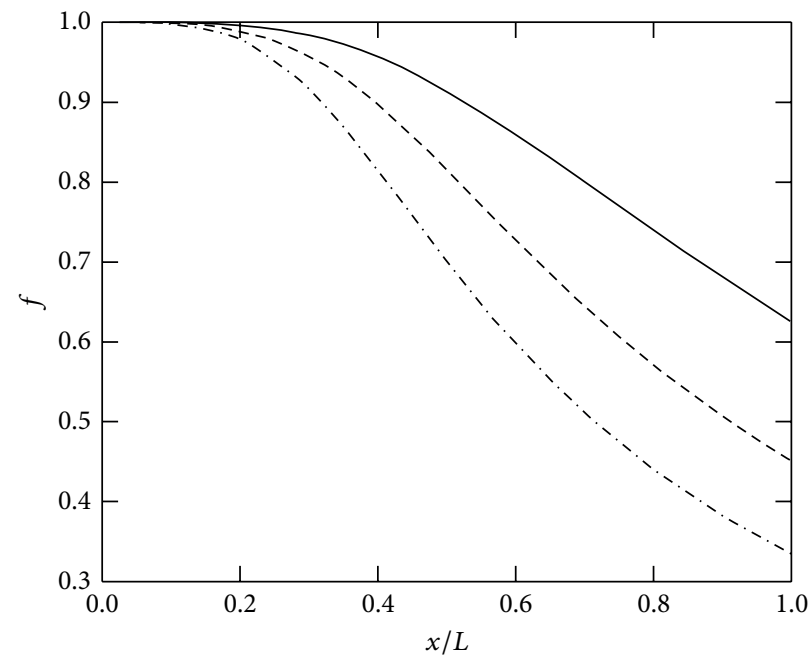

$$
\begin{array}{rl}
-m & =2 \\
--m & =5 \\
-\cdot m & m=10
\end{array}
$$

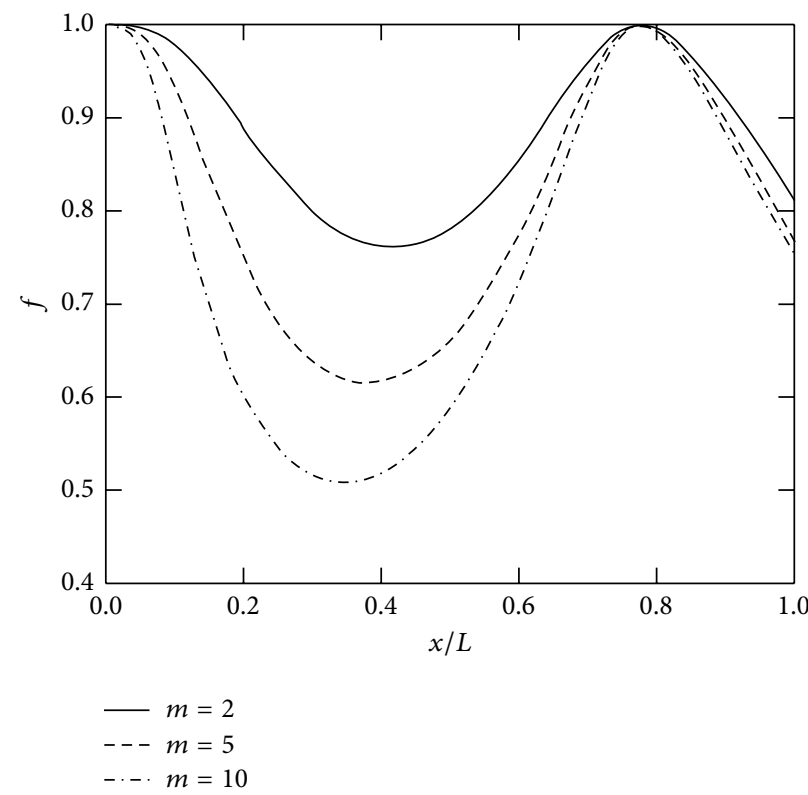

(b)

FIgURE 8: The first two normalized natural frequencies of clamped-simple Timoshenko beam subjected to a moving mass with different masses.

moving mass and cannot be neglected. Figure 10 shows that the tangential force is small than the others. The effect of the loads on the dynamic response of the Timoshenko beam is shown in Figure 11.

It shows that the displacements with the tangential force neglected agreed with the displacement without neglected any forces, because the property of the Timoshenko beam is that the ratio of the height to span is large; therefore, the deformation of the beam is smaller, and $y^{\prime}(\xi)$ is very small. The Coriolis force is the important role of the loads. From the formulations of the motion equations, the effect of Coriolis force is appearing in the damping matrix; hence, with Coriolis force neglected, the displacements are larger than those without any neglected forces. Thus, the effect of the Coriolis force should be considered. The effect of Centrifugal force and inertia force are appearing in the stiff and mass 

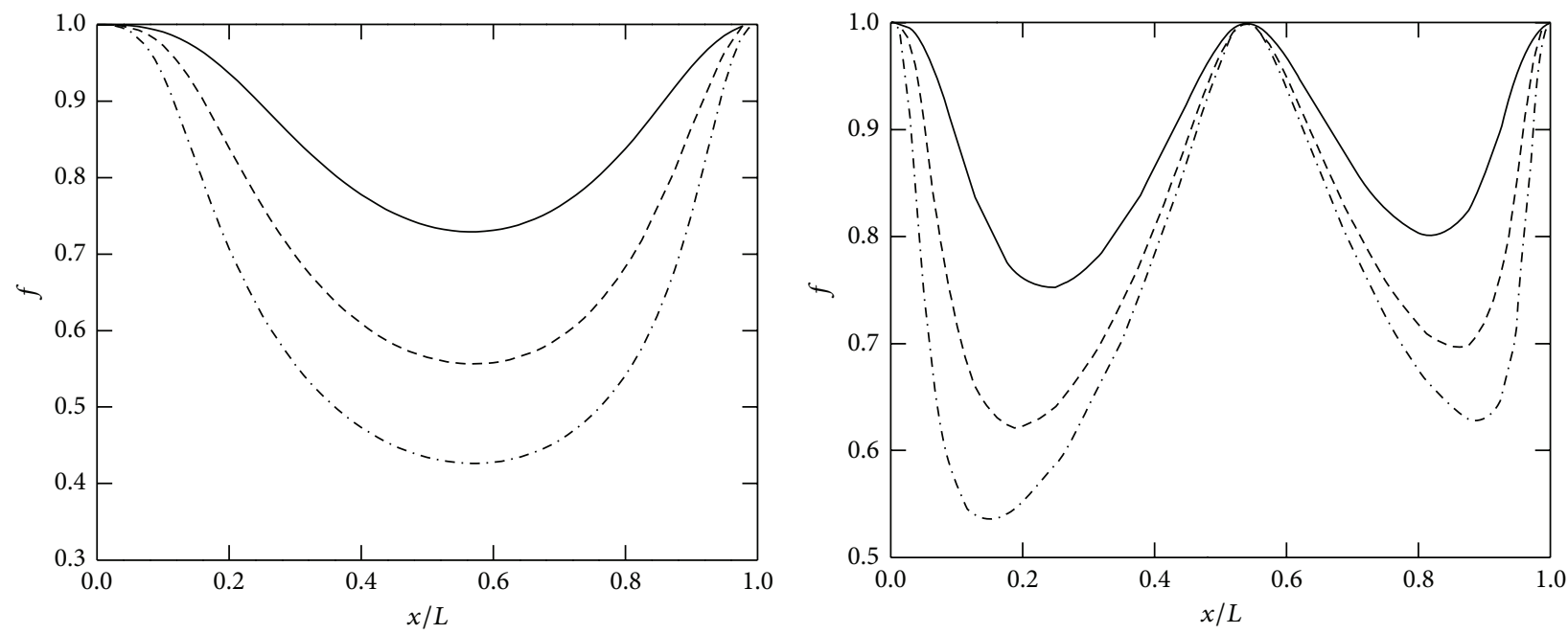

$\begin{aligned}-m & =2 \\ --m & =5 \\ --m & =10\end{aligned}$

$-m=2$

$--m=5$

-. $m=10$

(a)

(b)

Figure 9: The first two normalized natural frequencies of clamped-simple Timoshenko beam subjected to a moving mass with different masses.

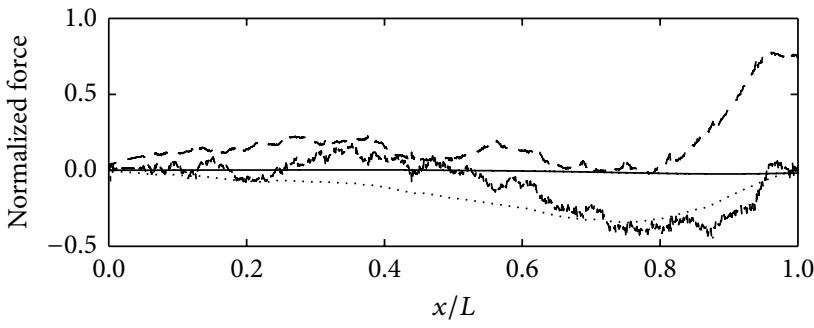

(a)

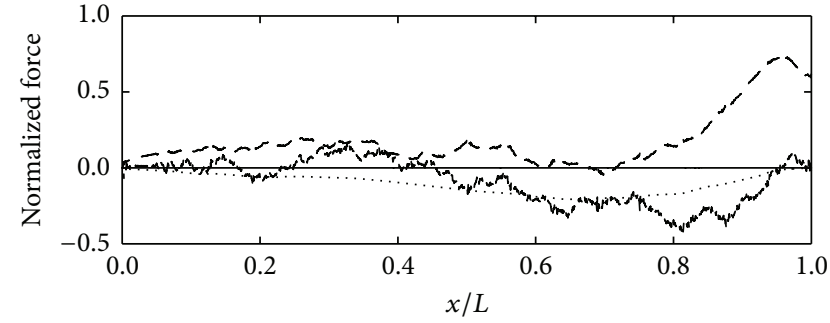

(b)

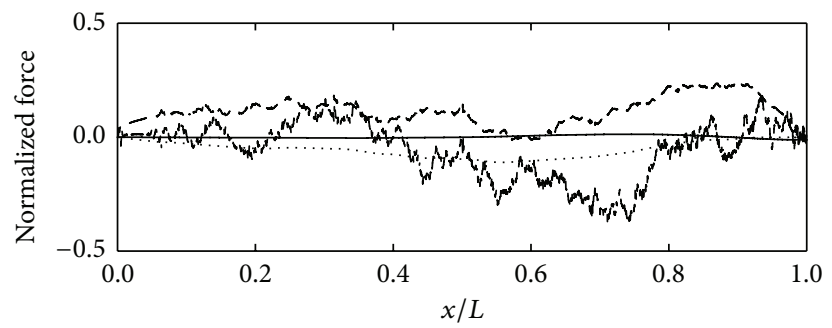

(c)

FIGURE 10: Normalized force under the equivalent moving loads with the initial speed given by $\tau=0.5 \cdot \beta=0.03$. (a) $\lambda=1$, (b) $\lambda=0$, and (c) $\lambda=-1$. Dashed line: inertia force; dotted dashed line: Coriolis force; dotted line: centrifugal force; solid line: tangential force.

matrix; therefore, with Centrifugal force and inertia being neglected, both the amplitude and the phase are different from those without any neglected forces.

\section{Conclusion}

The equation of motion in matrix form has been formulated for the dynamic response of a Timoshenko beam subjected to a moving mass by using TSEM. The inertia effect of the moving mass can easily be taken into account by assembling added mass, damping, and stiffness matrices to the global mass, damping, and stiffness matrices. The TSEM has the advantages of the spectral method and finite element method; the degrees of freedom can be less with the characteristic of high accuracy; the eigenvalue and dynamic problem can be obtained efficiently with the accurate diagonal mass matrix.

The variation of the vibration frequencies of the Timoshenko and a moving mass system was obtained. Numerical 


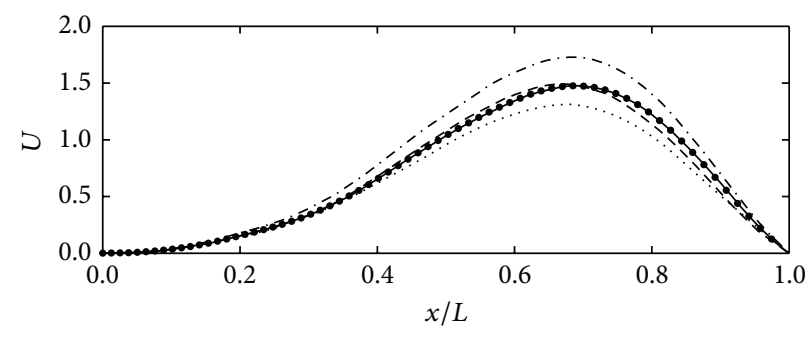

(a)

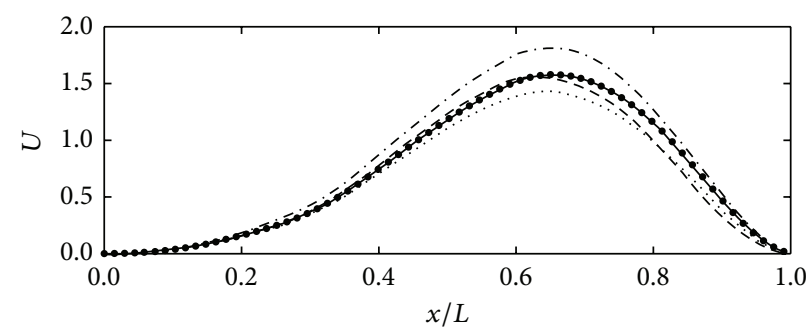

(b)

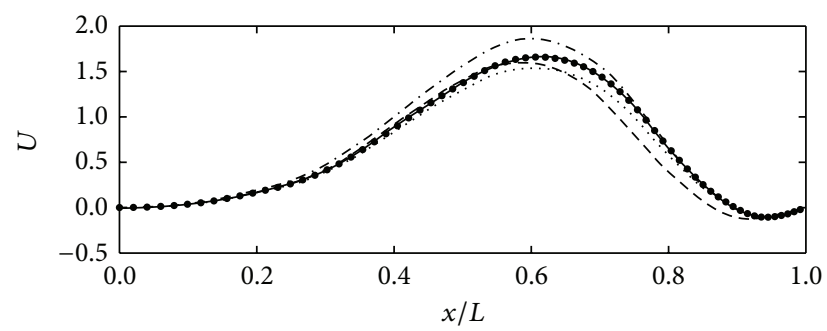

(c)

FIGURE 11: Normalized Displacement under the equivalent moving loads with the initial speed given by $\tau=0.5 \cdot \beta=0.03$. (a) $\lambda=1$, (b) $\lambda=0$, and (c) $\lambda=-1$. Solid line: without neglected; dashed line: with inertia force neglected; dotted dashed line: with Coriolis force neglected; dotted line: with centrifugal force neglected; bold dotted line: with tangential force neglected.

results for the Timoshenko beam and a moving mass system indicate that the inertia effect of the moving load cannot be neglected, the Coriolis, inertia, and centrifugal forces take a more important role than tangential force in the moving mass system, Coriolis, inertia, centrifugal, and tangential forces take the role of the damping, mass, and stiffness matrices, respectively.

\section{Conflict of Interests}

The authors declare that there is no conflict of interests regarding the publication of this paper.

\section{References}

[1] M. A. Foda and Z. Abduljabbar, "A dynamic green function formulation for the response of a beam structure to a moving mass," Journal of Sound and Vibration, vol. 240, no. 5, pp. 962970, 1998.

[2] J. Renard and M. Taazount, "Transient responses of beams and plates subject to travelling load," European Journal of Mechanics A, vol. 21, no. 2, pp. 301-322, 2002.

[3] E. Savin, "Dynamic amplification factor and response spectrum for the evaluation of vibrations of beams under successive moving loads," Journal of Sound and Vibration, vol. 248, no. 2, pp. 267-288, 2001.

[4] M. Abu-Hilal, "Dynamic response of a double Euler-Bernoulli beam due to a moving constant load," Journal of Sound and Vibration, vol. 297, no. 3-5, pp. 477-491, 2006.

[5] L. Fryba, Vibration of Solids and Structures under Moving Loads, Thomas Telford House, 1999.

[6] G. V. Rao, "Linear dynamics of an elastic beam under moving loads," Journal of Vibration and Acoustics, vol. 122, no. 3, pp. 281$289,2000$.
[7] H. S. Zibdeh and H. S. Juma, "Dynamic response of a rotating beam subjected to a random moving load," Journal of Sound and Vibration, vol. 223, no. 5, pp. 741-758, 1999.

[8] J. E. Akin and M. Mofid, "Numerical solution for response of beams with moving mass," Journal of Structural Engineering, vol. 115, no. 1, pp. 120-131, 1989.

[9] L. Sun, "Analytical dynamic displacement response of rigid pavements to moving concentrated and line loads," International Journal of Solids and Structures, vol. 43, no. 14-15, pp. 4370-4383, 2006.

[10] A. V. Pesterev, C. A. Tan, and L. A. Bergman, "A new method for calculating bending moment and shear force in moving load problems," Journal of Applied Mechanics, vol. 68, no. 2, pp. 252259, 2001.

[11] A. Greco and A. Santini, "Dynamic response of a flexural nonclassically damped continuous beam under moving loadings," Computers and Structures, vol. 80, no. 26, pp. 1945-1953, 2002.

[12] J. Hino, T. Yoshimura, K. Konishi, and N. Ananthanarayana, "A finite element method prediction of the vibration of a bridge subjected to a moving vehicle load," Journal of Sound and Vibration, vol. 96, no. 1, pp. 45-53, 1984.

[13] H. Ouyang and M. Wang, "Dynamics of a rotating shaft subject to a three-directional moving load," Journal of Vibration and Acoustics, vol. 129, no. 3, pp. 386-389, 2007.

[14] M. H. Kargarnovin, D. Younesian, D. J. Thompson, and C. J. C. Jones, "Response of beams on nonlinear viscoelastic foundations to harmonic moving loads," Computers and Structures, vol. 83, no. 23-24, pp. 1865-1877, 2005.

[15] P. Lou, G.-L. Dai, and Q.-Y. Zeng, "Dynamic analysis of a Timoshenko beam subjected to moving concentrated forces using the finite element method," Shock and Vibration, vol. 14, no. 6, pp. 459-468, 2007.

[16] A. S. J. Striker, R. de Borst, and C. Esveld, "Critical behaviour of a Timoshenko beam-half plane system under a moving load," Archive of Applied Mechanics, vol. 68, no. 3-4, pp. 158-168, 1998. 
[17] M. H. Kargarnovin and D. Younesian, "Dynamics of Timoshenko beams on Pasternak foundation under moving load," Mechanics Research Communications, vol. 31, no. 6, pp. 713-723, 2004.

[18] T. Kocatürk and M. Şimşek, "Dynamic analysis of eccentrically prestressed viscoelastic Timoshenko beams under a moving harmonic load," Computers and Structures, vol. 84, no. 31-32, pp. 2113-2127, 2006.

[19] J. W. Z. Zu and R. P. S. Han, "Dynamic response of a spinning Timoshenko beam with general boundary conditions and subjected to a moving load," Journal of Applied Mechanics, vol. 61, pp. 152-160, 1994.

[20] M. Şimşek, "Non-linear vibration analysis of a functionally graded Timoshenko beam under action of a moving harmonic load," Composite Structures, vol. 92, no. 10, pp. 2532-2546, 2010.

[21] T. J. Ross, "Dynamic rate effects on Timoshenko beam response," Journal of Applied Mechanics, vol. 52, no. 2, pp. 439445, 1985.

[22] R. Katz, C. W. Lee, A. G. Ulsoy, and R. A. Scott, "The dynamic response of a rotating shaft subject to a moving load," Journal of Sound and Vibration, vol. 122, no. 1, pp. 131-148, 1988.

[23] A. Yavari, M. Nouri, and M. Mofid, "Discrete element analysis of dynamic response of Timoshenko beams under moving mass," Advances in Engineering Software, vol. 33, no. 3, pp. 143-153, 2002.

[24] H. P. Lee, "The dynamic response of a Timoshenko beam subjected to a moving mass," Journal of Sound and Vibration, vol. 198 , no. 2, pp. 249-256, 1996.

[25] J. F. Doyle, Wave Propagation in Structures, Springer, New York, NY, USA, 1998.

[26] M. Krawczuk, M. Palacz, and W. Ostachowicz, "The dynamic analysis of a cracked Timoshenko beam by the spectral element method," Journal of Sound and Vibration, vol. 264, no. 5, pp. 1139-1153, 2003.

[27] D. Roy Mahapatra and S. Gopalakrishnan, "A spectral finite element model for analysis of axial-flexural-shear coupled wave propagation in laminated composite beams," Composite Structures, vol. 59, no. 1, pp. 67-88, 2003.

[28] M. Palacz and M. Krawczuk, "Analysis of longitudinal wave propagation in a cracked rod by the spectral element method," Computers and Structures, vol. 80, no. 24, pp. 1809-1816, 2002.

[29] A. T. Patera, "A spectral element method for fluid dynamics: laminar flow in a channel expansion," Journal of Computational Physics, vol. 54, no. 3, pp. 468-488, 1984.

[30] N. Azizi, M. M. Saadatpour, and M. Mahzoon, "Using spectral element method for analyzing continuous beams and bridges subjected to a moving load," Applied Mathematical Modelling, vol. 36, no. 8, pp. 3580-3592, 2012.

[31] G. Seriani, “3-D large-scale wave propagation modeling by spectral element method on Cray T3E multiprocessor," Computer Methods in Applied Mechanics and Engineering, vol. 164, no. 1-2, pp. 235-247, 1998.

[32] D. Komatitsch, C. Barnes, and J. Tromp, "Simulation of anisotropic wave propagation based upon a spectral element method," Geophysics, vol. 65, no. 4, pp. 1251-1260, 2000.

[33] C. Canuto, A. Quarteroni, M. Y. Hussaini, and T. A. Zang, Spectral Methods in Fluid Dynamics, Springer, Berlin, Germany, 1998.

[34] D. Komatitsch and J. Tromp, "Introduction to the spectral element method for three-dimensional seismic wave propagation," Geophysical Journal International, vol. 139, no. 3, pp. 806-822, 1999.
[35] H. Peng, G. Meng, and F. Li, "Modeling of wave propagation in plate structures using three-dimensional spectral element method for damage detection," Journal of Sound and Vibration, vol. 320, no. 4-5, pp. 942-954, 2009.

[36] S. Timoshenko, Vibration Problem in Engineering, 1937.

[37] J. Chung and G. M. Hulbert, "Time integration algorithm for structural dynamics with improved numerical dissipation: the generalized- $\alpha$ method," Journal of Applied Mechanics, vol. 60, no. 2, pp. 371-375, 1993.

[38] T. C. Huang, "The effect of rotatory inertia and of shear deformation on the frequency and normal mode equations of uniform beam with simple end conditions," Journal of Applied Mechanics, vol. 28, pp. 579-584, 1961.

[39] P. Ruta, "Application of Chebyshev series to solution of nonprismatic beam vibration problems," Journal of Sound and Vibration, vol. 227, no. 2, pp. 449-467, 1999. 

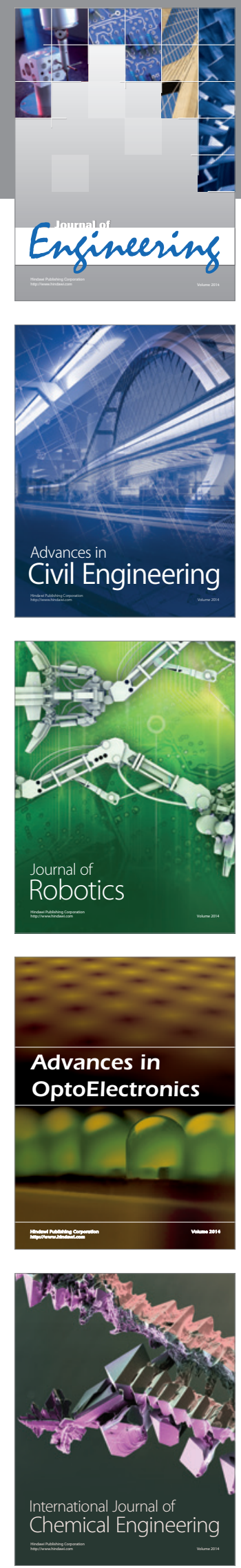

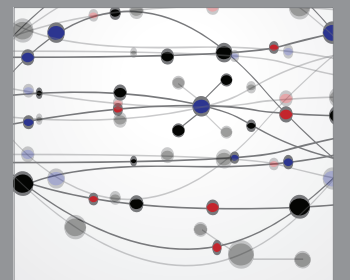

The Scientific World Journal
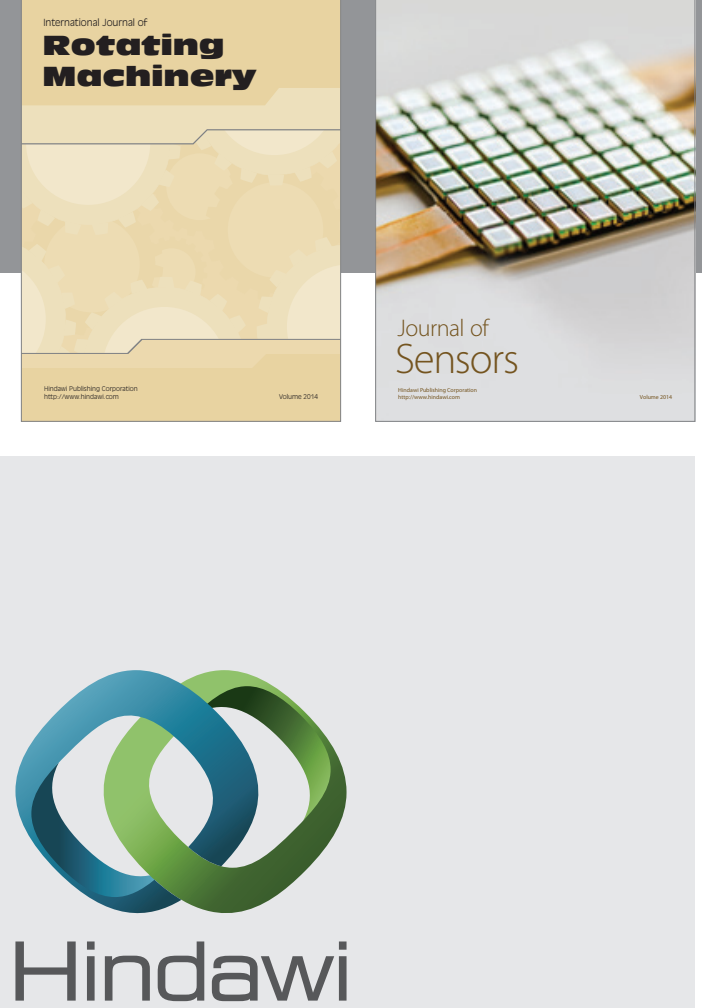

Submit your manuscripts at http://www.hindawi.com
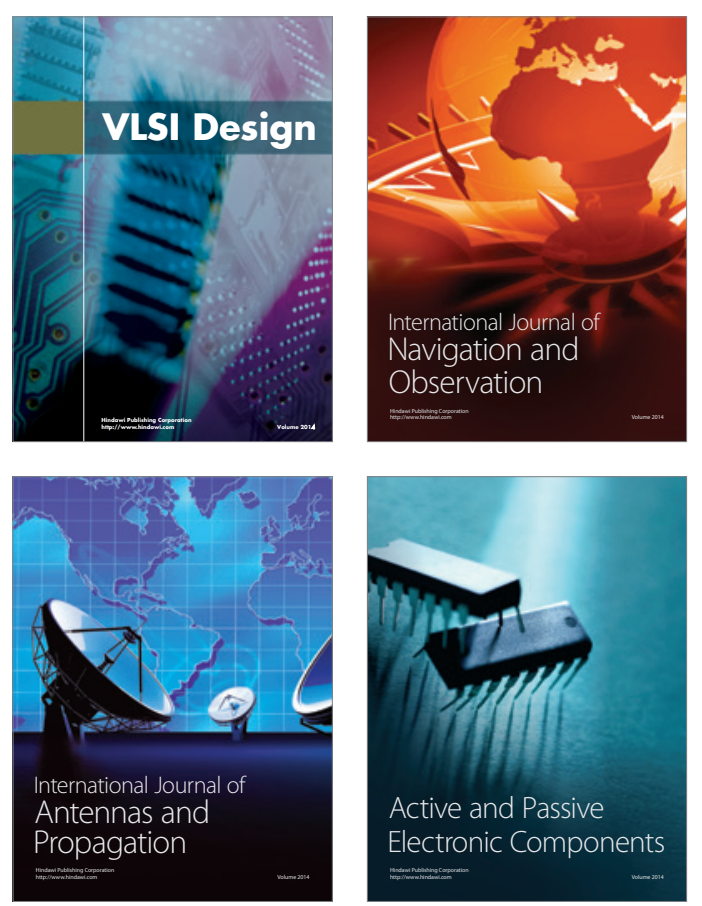
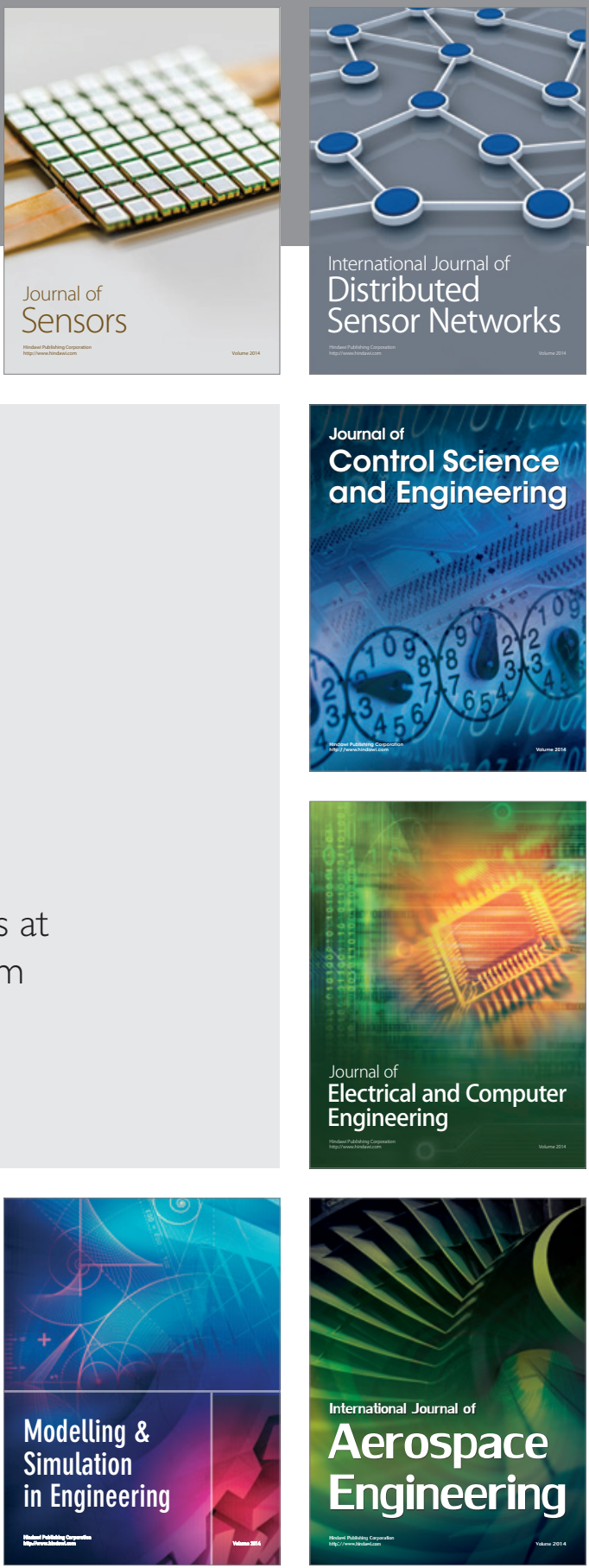

Journal of

Control Science

and Engineering
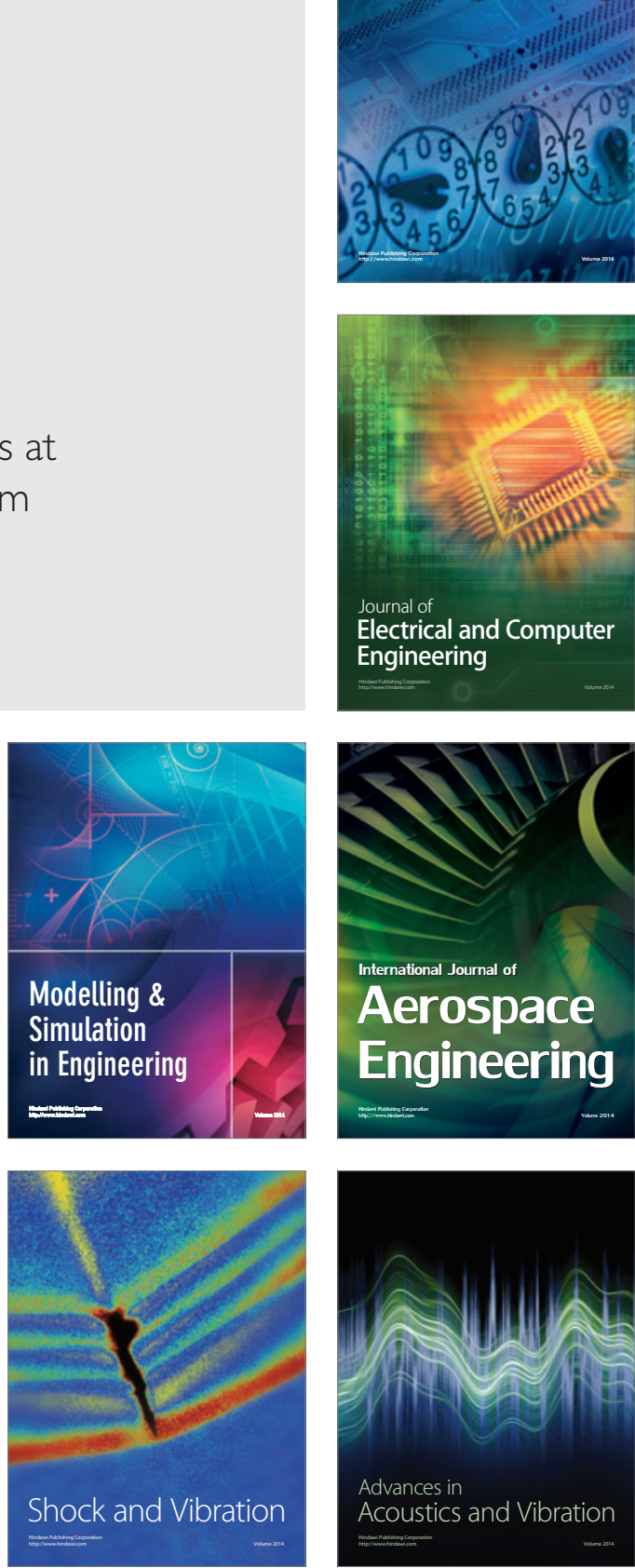